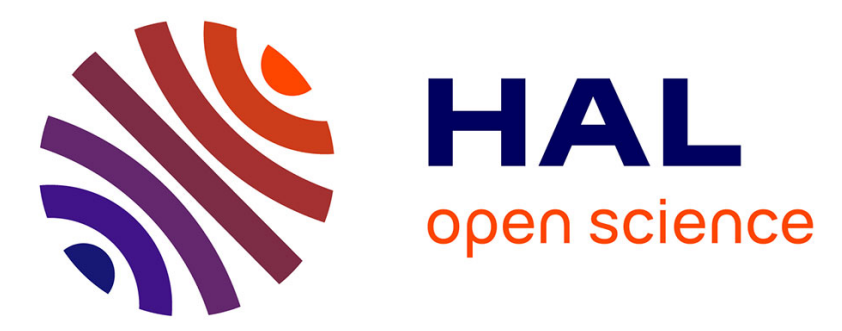

\title{
Analysis of a predator-prey model with specific time scales: a geometrical approach proving the occurrence of canard solutions
}

Jean-Christophe Poggiale, Clement Aldebert, Benjamin Girardot, Bob Kooi

\section{- To cite this version:}

Jean-Christophe Poggiale, Clement Aldebert, Benjamin Girardot, Bob Kooi. Analysis of a predatorprey model with specific time scales: a geometrical approach proving the occurrence of canard solutions. Journal of Mathematical Biology, 2020, 80 (1-2), pp.39-60. 10.1007/s00285-019-01337-4 . hal-03151454

\author{
HAL Id: hal-03151454 \\ https://hal.science/hal-03151454
}

Submitted on 24 Feb 2021

HAL is a multi-disciplinary open access archive for the deposit and dissemination of scientific research documents, whether they are published or not. The documents may come from teaching and research institutions in France or abroad, or from public or private research centers.
L'archive ouverte pluridisciplinaire HAL, est destinée au dépôt et à la diffusion de documents scientifiques de niveau recherche, publiés ou non, émanant des établissements d'enseignement et de recherche français ou étrangers, des laboratoires publics ou privés. 


\title{
Analysis of a predator-prey model with specific time scales : a geometrical approach proving the occurrence of canard solutions.
}

\author{
Poggiale Jean-Christophe · Aldebert Clément • \\ Girardot Benjamin - Kooi Bob.W.
}

Received: date / Accepted: date

\begin{abstract}
We study a predator-prey model with different characteristic time scales for the prey and predator populations, assuming that the predator dynamics is much slower than the prey one. Geometrical Singular Perturbation theory provides the mathematical framework for analyzing the dynamical properties of the model. This model exhibits a Hopf bifurcation and we prove that when this bifurcation occurs, a canard phenomenon arises. We provide an analytic expression to get an approximation of the bifurcation parameter value for which a maximal canard solution occurs. The model is the well-known Rosenzweig-MacArthur predator-prey differential system. An invariant manifold with a stable and an unstable branches occurs and a geometrical approach is used to explicitly determine a solution at the intersection of these branches. The method used to perform this analysis is based on Blow-up techniques. The analysis of the vector field on the blown-up object at an equilibrium point where a Hopf bifurcation occurs with zero perturbation parameter representing the time scales ratio, allows to prove the result. Numerical simulations illustrate the result and allow to see the canard explosion phenomenon.
\end{abstract}

Keywords Canard solution · Blow-up · Singular perturbations · Invariant manifolds · Predatorprey dynamics

Mathematics Subject Classification (2000) 92B05 $\cdot 34 \mathrm{C} 40 \cdot 37 \mathrm{G} 10 \cdot 37 \mathrm{~N} 25$

\section{Introduction}

Predation allows energy to flow across ecological communities and is as such an important process in ecosystem functioning and population dynamics. This has been the attention of a huge amount of works. Consequently, predator - prey models constitute a strong basis of current ecological theories. Among these models, the Rosenzweig-MacArthur model has been widely used since the exhibition of the Enrichment Paradox [42]. This model has been analyzed and extended (see [21] for instance). In this paper, we consider this model with two characteristic time scales associated to

\section{J.-C. POGGIALE}

Aix-Marseille Univ., Université de Toulon, CNRS, IRD, MIO UM 110, 13288, Marseille, France

E-mail: jean-christophe.poggiale@univ-amu.fr

C. ALDEBERT

Aix-Marseille Univ., Université de Toulon, CNRS, IRD, MIO UM 110, 13288, Marseille, France

B. GIRARDOT

Aix-Marseille Univ., Université de Toulon, CNRS, IRD, MIO UM 110, 13288, Marseille, France

B.W. KOOI

Faculty of Science, VU Amsterdam, De Boelelaan 1085, 1081 HV Amsterdam, The Netherlands. 
the prey and the predator populations. Natural systems involving several time scales are ubiquitous and taking care about time scales maybe crucial in management [17].

When time scales are taken into account explicitly in biological or ecological modelling, it may allow to develop methods for simplifying the mathematical analysis and understanding the dynamics of complex systems. A very common method is based on the Quasi-Steady State Approximation (QSSA) or time scale separation [45] [46], which has been extensively used in enzyme kinetics or biochemical systems, with more or less success [15]. The main principle is that if the vector of fast variables reaches an equilibrium, the corresponding variables are assumed to be almost constant and can be replaced by the equilibrium coordinates. Then only the slow variables are playing a role. From the mathematical point of view, this intuitive approach have been formalized in the singular perturbation theory [47], [20], [12], [48]. The interested reader can see [29] for a summary of methods and results in the study of slow-fast dynamics.

Geometrical Singular Perturbation theory (GSPT) has been developed on the basis of Fenichel's work [12]. In [23] and [50], general aspects of the theory are explained and illustrated with details and extensions. In [13], (see also [44]), Fenichel focuses his theorem to slow-fast dynamical systems and in [19], the interested reader can see for applications to biology. This theory provides methods to reduce the dimension of a set of differential equations involving several time scales by using invariant manifolds in the phase space. Reduction of the dynamics onto these invariant manifolds leads to decrease the dimension of the initial system to the dimension of the invariant manifolds, see [35] for an example where the power of GSPT is fully exploited with an accurate description of the dynamics of the complete model requiring an adapted representation of the invariant manifold. [2] presents the general approach in the context of ecological modelling. Singular perturbation methods have been used to build and analyze predator - prey systems (see [36], [37], [3], [38], [7] for instance) and have been proved to be efficient for explaining complex dynamics in food chains (e.g. [8], [9], [25], [34], [14]) or food webs [39].

Roughly speaking, Fenichel's theorem proves that a normally hyperbolic invariant manifold is persistent under small perturbations. It has been extended in [10] and [11] for situations where the normal hyperbolicity is lost (see also [27] and [49]). These papers propose a geometrical method of desingularization by blow-up of the singular points where the normal hyperbolicity is not satisfied, leading to explicitly build canard solutions. Canard phenomenon has been discovered in [4] and has been shown to occur in many systems with several time scales (e.g. [11], [26], [49], [31], [40], [22]).

In [24], we showed how to find a canard solution at the turning point in Rosenzweig-MacArthur model with two time scales by using asymptotic expansion methods. This paper completes the previous one by proving that such a canard solution exists and by providing an explicit construction by means of blow-up technique. The turning point is a particular case of the general developments proposed in [10], [11] and [27]. This paper is an illustration of their method on a very common predator-prey model. Since the canard solutions occur after a Hopf bifurcation, we provide an explicit algebraic relationship between the bifurcation parameter and the small parameter representing the time scale ratio. As it can be seen in [10], [11] and [27], the method is general and can be used for other models. Moreover, in Rosenzweig-MacArthur model, another canard solution occurs at the point located at the intersection of the prey isocline and the vertical axis. This point corresponds to a transcritical bifurcation of the fast system and is called a transcritical singularity (see [28] for instance). It can also dealt with a blow-up approach as we illustrate in the appendix of this paper. We discuss this further in the paper.

Predator-prey models with two time scales have already be the topics of several papers and slowfast limit cycles have already been highlighted in these models (see [41] for instance). Moreover, canard phenomenons in predator - prey systems have already be mentioned or conjectured in previous works (see [1], [19], or [32] for instance) but never been analyzed in detail with a generic method. Note that in dimension 2, this is more a mathematical curiosity than an ecological feature. However, the method used here is general and can be applied to more realistic situations. In higher dimension (e.g. food chain), the method still applies and can actually explain different types of fluctuations (as mixed-mode oscillations, see for instance [6] or [43]). In this case, the canard phenomenon would help to understand food chain dynamics and then contribute to explain ecological properties. 
The paper is organised as follows. In section 2, the model is presented and time scales are introduced. Notations and definitions are provided in the following section and some well-known results are recalled. Section 3 states the main result and the proof follows. Phase portraits are shown for bifurcation parameter values close to where a canards explosion occurs in subsections of 3 . Section 4 concludes the paper with discussions and conclusions. The appendix deals with the blow-up of the transcritical singularity.

\section{The model: description and generalities}

\subsection{Model description}

Let us consider the following model (Rosenzweig - MacArthur) :

$$
\begin{aligned}
& \frac{d x}{d t}=r x\left(1-\frac{x}{K}\right)-\frac{a x}{b+x} y \\
& \frac{d y}{d t}=\left(e^{\prime} \frac{a x}{b+x} y-m^{\prime} y\right)
\end{aligned}
$$

and we assume that the parameters $e^{\prime}$ and $m^{\prime}$ are small, so that the dynamics of the predator is slow with respect to the prey dynamics. In order to make this explicit, we define $\varepsilon$ a small positive dimensionless parameter and we set $e^{\prime}=\varepsilon e$ and $m^{\prime}=\varepsilon m^{\prime}$ where $e$ and $m$ are rescaled conversion efficiency and mortality rate. The model reads :

$$
\begin{aligned}
& \frac{d x}{d t}=r x\left(1-\frac{x}{K}\right)-\frac{a x}{b+x} y \\
& \frac{d y}{d t}=\varepsilon\left(e \frac{a x}{b+x} y-m y\right)
\end{aligned}
$$

We shall now eliminate some parameters by nondimensionalization for simplifying the mathematical study of the model. Let $x_{1}=\frac{x}{K}, y_{1}=\frac{y}{e K}$ and $t_{1}=r t$ be the new variables and $a_{1}=\frac{e a}{r}$, $b_{1}=\frac{b}{K}$ and $m_{1}=\frac{m}{r}$ be the new parameters. We replace the variables and parameters in the previous model and we omit the index 1 in all symbols for keeping notations simple. The new model reads:

$$
\begin{aligned}
& \frac{d x}{d t}=x\left(1-x-\frac{a}{b+x} y\right) \\
& \frac{d y}{d t}=\varepsilon\left(\frac{a x}{b+x}-m\right) y
\end{aligned}
$$

Finally, we make a last transformation in order to get a polynomial vector field. This transformation is not necessary for the method to apply, we just use it for convenience. Accordingly, since we restrict our study to the positive domain $K^{+}=\{(x, y) \mid \geq 0, y \geq 0\}$, we can multiply the vector field defined by the previous differential system by the positive factor $b+x$, this does not change the trajectories in the phase space. Consequently, we now study the following system:

$$
\begin{aligned}
& \frac{d x}{d t}=x\left(b+(1-b) x-a y-x^{2}\right) \\
& \frac{d y}{d t}=\varepsilon((a-m) x-m b) y
\end{aligned}
$$

We assume in this paper that $a>m$, that is the predator maximal growth rate is larger that its death rate. 


\subsection{General results and notations}

Rosenzweig-MacArthur's model has already been studied and the dynamics is well known. We briefly summarize some results here because they will be useful in the following section.

System (20) leaves the set $K^{+}$invariant. In this set, the prey isoclines are the vertical axis $\{x=0\}$ and the parabola defined by $y=\frac{(1-x)(b+x)}{a}$. The predator isoclines are the horizontal axis $\{y=0\}$ and the vertical straight line $\left\{x=\frac{m b}{a-m}\right\}$. Let us consider the assumption :

$$
\max \left(b, \frac{m b}{a-m}\right)<1 \text {. }
$$

Under assumption (2), the vertical predator isocline crosses the previous parabola in $K^{+}$and there exists a positive equilibrium, which will be denoted by $E=\left(x_{E}, y_{E}\right)$ with $x_{E}=\frac{m b}{a-m}$ and $y_{E}=\frac{\left(1-x_{E}\right)\left(b+x_{E}\right)}{a}$. Let us also denote by $S_{T}$ the top of the previous parabola, its coordinates are $S_{T}=\left(x_{T}, y_{T}\right)=\left(\frac{1-b}{2}, \frac{(1+b)^{2}}{4 a}\right)$. Assumption (2) is satisfied in the entire paper.

Moreover, if the vertical predator isoclines crosses the parabola on the right of $S_{T}$ then $E$ is globally asymptotically stable in the interior of $K^{+}$and if the intersection is on the left of $S_{T}$ then $E$ is unstable and is surrounded by a unique limit cycle in $K^{+}$. In this case, the limit cycle attracts all trajectories starting in the interior of $K^{+}$but $E$. Indeed, when the vertical predator isocline moves and crosses the top of the parabola, a Hopf bifurcation occurs.

Since we consider two time scales in this paper, the parameter $\varepsilon$ is small and we use perturbation theory to see the shape of the trajectories when $\varepsilon$ is very close to zero but not null. Let us start with the extreme case where $\varepsilon=0$. Then $y$ is a constant and the dynamics of $x$ vanishes on the vertical axis $\{x=0\}$ and on the parabola $\left\{y=\frac{1}{a}(b+x)(1-x)\right\}$, which, as sets of equilibria, are invariant manifolds denoted $\mathcal{M}_{10}$ and $\mathcal{M}_{20}$ respectively. The intersection between the vertical axis $\mathcal{M}_{10}$ and the parabola $\mathcal{M}_{20}$ is the point $S_{C}=\left(x_{C}, y_{C}\right)=\left(0, \frac{b}{a}\right)$. The maximum of the parabola $\mathcal{M}_{20}$ is the point $S_{T}$.

At these points, the invariant sets are not normally hyperbolic : $\mathcal{M}_{10}$ loses the normal hyperbolicity at $S_{C}$ and $\mathcal{M}_{20}$ loses the normal hyperbolicity at $S_{C}$ and $S_{T}$. $S_{T}$ is called a fold point because when $\varepsilon=0$ and $y$ increases and crosses the $y$-value of $S_{T}$, a fold bifurcation takes place. Geometrical Singular Perturbation Theory (GSPT), initiated by Fenichel's work, provides mathematical results for analyzing the dynamics around invariant manifolds when they are normally hyperbolic. Extension methods have been provided for singular points on the invariant manifolds where the normal hyperbolicity is lost, which correspond to a bifurcation of the fast dynamics. Among them, the blow-up technique allows to build a new geometrical object and a new vector field on this object, by change of variables, such that for the new system, the invariant manifolds are normally hyperbolic. This is a so-called desingularization method. In this works, we apply this approach to study the dynamics of the Rosenzweig-MacArthur model with two time scales around the singular points $S_{T}$.

The next section is devoted to the mathematical analysis of system (20) in order to show that when the Hopf bifurcation takes place, a canard solution occurs. We then describe the dynamics of the system in the phase space for small positive values of $\varepsilon$ around the Hopf bifurcation.

\section{Canard solutions in the Rosenzweig - MacArthur model with a geometrical method}

\subsection{Brief description of the blow-up method, notations and definitions}

In this section, we simplify the study by change of variables and explain briefly how the blowup method works. Then we provide some notations and definitions. We start by making a new 
change of variables in order to move the positive equilibrium $E$ to the origin. Note that often, when analyzing fold points like $S_{T}$, the translation is made such that the fold point is moved to the origin. Actually, here, it does not matter because as we will analyse the Hopf bifurcation at this point, $S_{T}$ and $E$ coincide at the bifurcation. Then our change of coordinates is efficient to understand the bifurcation and the occurrence of a canard phenomenon. Moreover, we denote by $\lambda$ the bifurcation parameter, which is defined by $\lambda=1-b-2 x_{E}$, so that the bifurcation occurs for $\lambda=0$. We then replace the parameter $a$ by its expression with respect to $\lambda$, that is $a=m \frac{1+b-\lambda}{1-b-\lambda}$

Let $X=x-x_{E}$ and $Y=y-y_{E}$ and $\lambda$ defined as above, system (20) then reads:

$$
\begin{aligned}
\frac{d X}{d t} & =\left(X+x_{E}\right)\left(\lambda X-m \frac{1+b-\lambda}{1-b-\lambda} Y-X^{2}\right) \\
\frac{d Y}{d t} & =\varepsilon \frac{2 b m}{1-b-\lambda} X\left(Y+y_{E}\right)
\end{aligned}
$$

We consider this system on the set $\tilde{K}^{+}=\left\{(X, Y) \mid X \geq-x_{E}, Y \geq-y_{E}\right\}$. Note that if $b>1$, then $\lambda$ would always be negative. Thus the Hopf bifurcation can occur only when $b<1$, we make this assumption from now. In differential system (3), there are 4 parameters. We consider $\lambda$ close to 0 , and $\lambda_{c}=0$ is a bifurcation value for this parameter. Finally, note that $x_{E}$ and $y_{E}$ are combinations of parameters depending on $\lambda$, such that they are strictly positive for $\lambda=0$.

From now, system (3) will be considered as a differential system defining a family of vector fields $\mathcal{X}_{\mu}$ on $\tilde{K}^{+}$where $\mu=(\varepsilon, \lambda) \in \Lambda$ with $\Lambda=\left[0, \varepsilon_{0}\right) \times I$ where $I$ is a small interval containing 0 and $\varepsilon_{0}>0$. In this family, the point $(0,0,0,0) \in K^{+} \times \Lambda$ is a so called non-degenerate singular fold point. As we previously said, Fenichel's theorem does not apply because of the loss of normal hyperbolicity. In [10] and [11], the authors develop the general approach for analyzing singular fold points in dynamical systems with two time scales. In [27], the authors address the study of non-degenerate singular fold points and extend the previous results. Our case is thus a particular case of this general study.

To analyze this singular fold point, we complete system (3) by the equations $\frac{d \varepsilon}{d t}=0$ and $\frac{d \lambda}{d t}=0$. We thus get a vector field in the vicinity of 0 in a 4-dimensional space, which leaves all the planes $\mathcal{P}_{u, v}=\{(X, Y, \varepsilon, \lambda) \mid \varepsilon=u, \lambda=v\}$ invariant, where $u$ and $v$ are any real number in a vicinity of 0 .

As we already reminded, for all $\lambda \in I$, the parabola defined by $Y=\frac{(1-b-\lambda)}{m(1+b-\lambda)}\left(\lambda X-X^{2}\right)$ is invariant under the flow of $\mathcal{X}_{\mu}$ when $\varepsilon=0$. The union of all these parabolas constitutes an invariant 3-dimensional manifold for $\varepsilon=0$ that we denote by $\tilde{\mathcal{M}}_{20}$. We define by $\tilde{\mathcal{M}}_{20}^{S}$ the stable branch of the invariant manifold and $\tilde{\mathcal{M}}_{20}^{U}$ is the unstable branch. The former is the part of the manifold contained in the subset where $X>0$ and $\varepsilon \geq 0$ and the latter is the part of the manifold contained in the subset where $X<0$ and $\varepsilon \geq 0$, see Figure 1 . For $\varepsilon=0$, the stable branch and the unstable branch of the manifold are connected. According to Fenichel's theorem, when the invariant manifold $\mathcal{M}_{20}$ is normally hyperbolic, it persists for small positive values of $\varepsilon$. In other words, there exists an invariant manifold $\mathcal{M}_{2 \varepsilon}^{S}$ close to $\mathcal{M}_{20}^{S}$ and an invariant manifold $\mathcal{M}_{2 \varepsilon}^{U}$ close to $\mathcal{M}_{20}^{U}$. We now have the elements to more precisely define a canard solution.

Definition 1 A solution of system (3) lying in the intersection between the stable branch and the unstable branch of $\mathcal{M}_{2 \varepsilon}$ is called a maximal canard.

Our aim in this paper is to prove that such a solution exists and the consequences on the dynamical properties of Rosenzweig-MacArthur model. In order to show that a canard phenomenon occurs in model (3), we use a blow-up approach. A blow-up is a geometrical transformation of the phase space around a singular point such that in the new geometrical object, the singular point is desingularized allowing the mathematical analysis. Note that in this paper, we focus the analysis on the canard solution arising at the top of the parabola, but in the Rosenzweig-MacArthur, another canard solution takes also place at the transcritical point located on the intersection of 
(A)

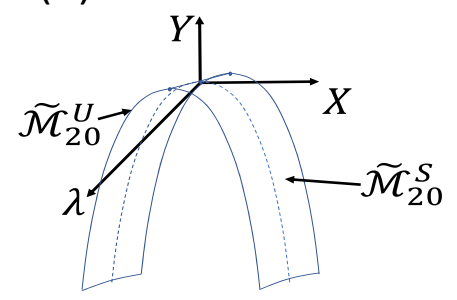

(B)

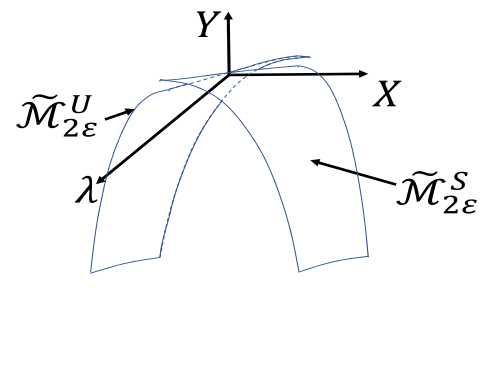

Fig. 1 Scheme of the invariant manifolds $\mathcal{M}_{2 \varepsilon}$ for $\varepsilon=0$ (A) and for $\varepsilon>0$ and small (B).

the parabola with the vertical axis. This set of two canard phenomenons in the same differential system is known as a canard doublet (see [40]). This is an important feature which leads to relaxation oscillations in the model. It may be analyzed with the blow-up approach as well (see Appendix A for some details). The canard solution arising at the transcritical point leads to a so called delayed bifurcation (see [5], [16] or [49] for instance) because trajectories approaching such a point along the stable part of the vertical axis will remain a finite time along the unstable part of the vertical axis before leaving (see [34] or [49] for instance).

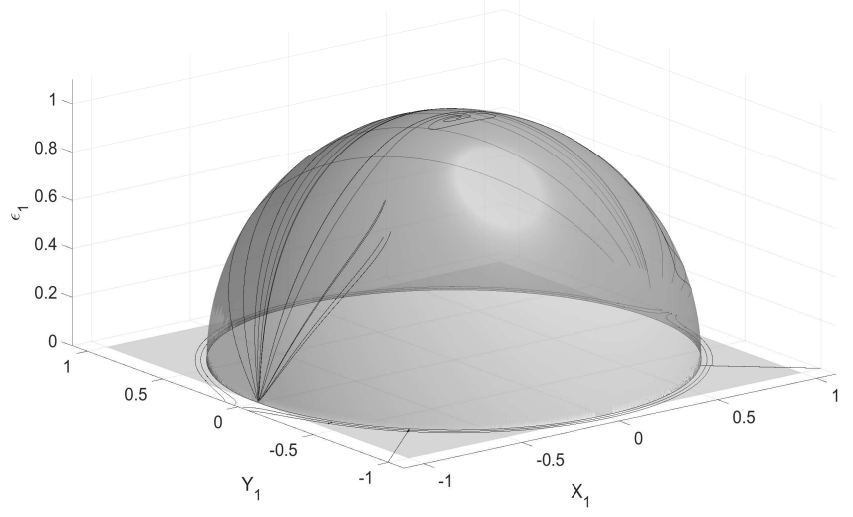

Fig. 2 Scheme of blow-up around the singularity $(0,0,0,0)$ for a fixed positive $\lambda$.

We desingularize the point $(0,0,0,0) \in \tilde{K}^{+} \times \Lambda$ by considering the blow-up :

$$
\begin{aligned}
& \Psi: S^{3} \times\left[0,+\infty\left[\longrightarrow \mathbb{R}^{4}\right.\right. \\
& \left(X_{1}, Y_{1}, \lambda_{1}, \varepsilon_{1}, r\right) \mapsto(X, Y, \varepsilon, \lambda)=\left(r X_{1}, r^{2} Y_{1}, r^{2} \varepsilon_{1}, r \lambda_{1}\right)
\end{aligned}
$$

where

$$
X_{1}^{2}+Y_{1}^{2}+\lambda_{1}^{2}+\varepsilon_{1}^{2}=1
$$

Figure 2 illustrates the blow-up result. The singular fold point has been replaced by a hemisphere. The vector field on the horizontal set $\{\varepsilon=0\}$ has been determined from analysis in charts 
( $\left\{X_{1}= \pm 1\right\}$ and $\left\{Y_{1}= \pm 1\right\}$ (see details below) and then projected on the hemisphere equator (circle). This projection is done as follows. For each chart in the set $\{\varepsilon=0\}$, we can consider that there is an axis tangent to the circle and an axis in the radial direction. We use the Poincaré compactification of the tangent axis which projects this tangent axis onto half of the circle and the radial direction is projected such that the radial distances are conserved. The vector field on the hemisphere is determined by the analysis in the chart $\{\varepsilon=1\}$ and then projected on the sphere by Poincaré compactification.

We then start with a vector field defined on the vicinity of $0 \in \tilde{K}^{+} \times \Lambda$ and we build a vector field on a geometrical object where the point 0 is replaced by a hemisphere (because $\varepsilon \geq 0$ ).

Firstly we write the set of ODEs after the change of coordinates where $r(t)$ is assumed to be a function of time.

$$
\begin{aligned}
\frac{d X_{1}}{d t} & =\frac{1}{r}\left[\frac{d X}{d t}-X_{1} \frac{d r}{d t}\right] \\
\frac{d Y_{1}}{d t} & =\frac{1}{r^{2}}\left[\frac{d Y}{d t}-2 r Y_{1} \frac{d r}{d t}\right] \\
\frac{d \varepsilon_{1}}{d t} & =-\frac{2 \varepsilon_{1}}{r} \frac{d r}{d t} \\
\frac{d \lambda_{1}}{d t} & =-\frac{\lambda_{1}}{r} \frac{d r}{d t}
\end{aligned}
$$

where $\frac{d X}{d t}$ and $\frac{d Y}{d t}$ are given in (3). In order to understand the dynamics on this hemisphere, we use charts. For instance, the chart $\left\{X_{1}=1\right\}$ describes the dynamics of the new vector field around the hemisphere (denoted by $S^{3+}$ ) for positive $X_{1}$, the chart $\left\{X_{1}=-1\right\}$ describes the dynamics around the hemisphere for negative $X_{1}$ and so on with the charts $\left\{Y_{1}=1\right\},\left\{Y_{1}=-1\right\}$ and $\left\{\varepsilon_{1}=1\right\}$. We first detail the chart $\left\{X_{1}=1\right\}$ for explaining the method. In the proof of theorem (1), we will just give the results for the other chart but we will provide more details for the charts $\left\{\varepsilon_{1}=1\right\}$ (for negative, null and positive $\lambda$ ) because it allows to understand the birth of the canard phenomenon explicitly.

To get the chart $\left\{X_{1}=1\right\}$, we consider the change of coordinates $(X, Y, \varepsilon, \lambda)=\left(r, r^{2} Y_{1}, r^{2} \varepsilon_{1}, r \lambda_{1}\right)$ which leads to:

$$
\begin{aligned}
\frac{d r}{d t} & =\frac{d X}{d t} \\
\frac{d Y_{1}}{d t} & =\frac{1}{r^{2}}\left[\frac{d Y}{d t}-2 r Y_{1} \frac{d r}{d t}\right] \\
\frac{d \varepsilon_{1}}{d t} & =-\frac{2 \varepsilon_{1}}{r} \frac{d r}{d t} \\
\frac{d \lambda_{1}}{d t} & =-\frac{\lambda_{1}}{r} \frac{d r}{d t}
\end{aligned}
$$

This is a special case of system (4) with $\frac{d X_{1}}{d t}=0$ because $X_{1}=1$ hence $X=r$. After some straighforward calculations, assuming that $r$ is small and expanding the equations with respect to $r$, one gets:

$$
\begin{aligned}
\frac{d r}{d t} & =r^{2} \frac{1-b}{2}\left(-1+\lambda_{1}-m \frac{1+b}{1-b} Y_{1}\right)+O\left(r^{3}\right) \\
\frac{d Y_{1}}{d t} & =r\left(b \frac{1+b}{2} \varepsilon_{1}-Y_{1}(1-b)\left(\lambda_{1}-1-m \frac{1+b}{1-b} Y_{1}\right)\right)+O\left(r^{2}\right)
\end{aligned}
$$

Let us consider the case $\varepsilon_{1}=0$ and $\lambda_{1}=0$ in the previous system, and after division by $r$, one gets:

$$
\begin{aligned}
\frac{d r}{d t} & \left.=r \frac{1-b}{2}\left(-1-m \frac{1+b}{1-b} Y_{1}\right)\right)+O\left(r^{2}\right) \\
\frac{d Y_{1}}{d t} & =-Y_{1}(1-b)\left(-1-m \frac{1+b}{1-b} Y_{1}\right)+O(r)
\end{aligned}
$$


(A)

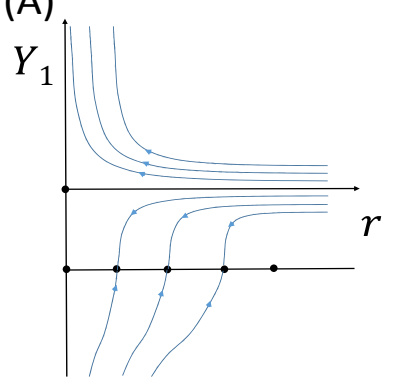

(B)

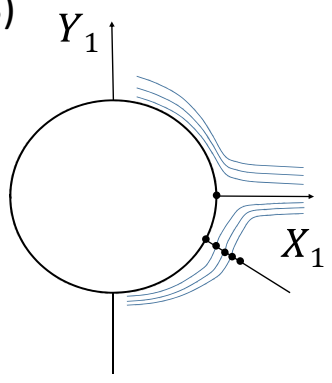

Fig. 3 Scheme of the dynamics in a chart $\left\{X_{1}=1\right\}$ for $\varepsilon_{1}=0$ and $\lambda_{1}=0$, given by system (7) (panel (A)). Panel (B) illustrates the transformation of the dynamics around the hemisphere in the plane $\varepsilon_{1}=0$ and $\lambda_{1}=0$, provided by the study in the previous chart $\left\{X_{1}=1\right\}$. Black points correspond to equilibria.

Note that dividing by $r$ for $r>0$ does not change the trajectories and allows to determine the dynamics around the hemisphere and this is why we can desingularize the origin. The study of the dynamics is simple and the main results are that the vertical axis is invariant as well as the straight line defined by $Y_{1}=-\frac{1-b}{m(1+b)}$. These invariant sets in the chart $\left\{X_{1}=1\right\}$ correspond to invariant sets on the blown-up geometrical object, namely respectively to the hemisphere equator (circle) and to the stable branch of the parabola (straight lines perpendicular to the circle), in the plane $\left\{\varepsilon_{1}=0\right\} \bigcap\left\{\lambda_{1}=0\right\}$. Results are illustrated in Figure 3 .

\subsection{Main results}

Theorem 1 For $\varepsilon>0$ small enough, system (20) admits maximal canard solutions when $\lambda$ becomes positive and close to zero. More precisely, there exists a function defined in the vicinity of $0 \in \mathbb{R}$ with $\varepsilon \mapsto \lambda_{1 c}(\varepsilon)$ such that for all $\varepsilon>0$ close to 0 , there exists $\lambda=\lambda_{c}(\varepsilon)>0$ for which system (20) exhibits a maximal canard. An approximation of this function is provided by:

$$
\lambda_{c}(\varepsilon)=\frac{m b(1+b)^{2}}{(1-b)^{3}} \varepsilon+O\left(\varepsilon^{3 / 2}\right)
$$

Proof The proof mainly rests on the blow-up of the fold point. We first complete the blow-up with all the needed charts and focus on the study in chart $\{\varepsilon=1\}$ for different values of $\lambda$ around 0 .

In chart $\left\{X_{1}=-1\right\}$, and after division by $r$, the vector field around the hemisphere is provided by:

$$
\begin{aligned}
\frac{d r}{d t} & \left.=r \frac{1-b}{2}\left(\lambda_{1}+1+m \frac{1+b}{1-b} Y_{1}\right)\right)+O\left(r^{2}\right) \\
\frac{d Y_{1}}{d t} & =-\varepsilon_{1} b \frac{1+b}{2}-Y_{1} r(1-b)\left(1+\lambda_{1}+Y_{1} m \frac{1+b}{1-b}\right)+O(r) \\
\frac{d \varepsilon_{1}}{d t} & =\varepsilon_{1}(1-b)\left(1+\lambda_{1}+m Y_{1} \frac{1+b}{1-b}\right)+O(r) \\
\frac{d \lambda_{1}}{d t} & =-\lambda_{1} \frac{1-b}{2}\left(1+\lambda_{1}+m \frac{1+b}{1-b}\right)+O(r)
\end{aligned}
$$

Note that in the plane $\varepsilon_{1}=0$ and $\lambda_{1}=0$, this system leaves the straight line defined by $Y_{1}=$ $-\frac{1-b}{m(1+b)}$ invariant. This straight line corresponds to the unstable branch of the parabola in the initial blow-up geometrical object. 

by:

In chart $\left\{Y_{1}=1\right\}$, and after division by $r$, the vector field around the hemisphere is provided

$$
\begin{aligned}
\frac{d X_{1}}{d t} & =\frac{1-b}{2}\left(\lambda_{1} X_{1}-m \frac{1+b}{1-b}-X_{1}^{2}\right)-\varepsilon_{1} \frac{(1+b) b}{4} X_{1}^{2}+O(r) \\
\frac{d r}{d t} & =r \varepsilon_{1} \frac{(1+b) b}{4} X_{1}+O\left(r^{2}\right) \\
\frac{d \varepsilon_{1}}{d t} & =-2 \varepsilon_{1}^{2} \frac{(1+b) b}{4} X_{1}+O(r) \\
\frac{d \lambda_{1}}{d t} & =-\lambda_{1} \varepsilon_{1} \frac{(1+b) b}{4} X_{1}+O(r)
\end{aligned}
$$

In chart $\left\{Y_{1}=-1\right\}$, and after division by $r$, the vector field around the hemisphere is provided by:

$$
\begin{aligned}
\frac{d X_{1}}{d t} & =\frac{1-b}{2}\left(\lambda_{1} X_{1}+m \frac{1+b}{1-b}-X_{1}^{2}\right)+\varepsilon_{1} \frac{(1+b) b}{4} X_{1}^{2}+O(r) \\
\frac{d r}{d t} & =-r \varepsilon_{1} \frac{(1+b) b}{4} X_{1}+O\left(r^{2}\right) \\
\frac{d \varepsilon_{1}}{d t} & =-2 \varepsilon_{1}^{2} \frac{(1+b) b}{4} X_{1}+O(r) \\
\frac{d \lambda_{1}}{d t} & =-\lambda_{1} \varepsilon_{1} \frac{(1+b) b}{4} X_{1}+O(r)
\end{aligned}
$$

Note that in the plane $\varepsilon_{1}=0$ and $\lambda_{1}=0$, this system leaves the straight lines defined by $X_{1}= \pm \sqrt{\frac{m(1+b)}{1-b}}$ invariant. These straight lines correspond to the stable and unstable branches of the parabola. Indeed, it can be noticed that on these lines, $X_{1}^{2}=\frac{m(1+b)}{1-b}$ which is the negative reciprocal of $Y_{1}$ on the invariant straight lines in charts $\left\{X_{1}= \pm 1\right\}$.

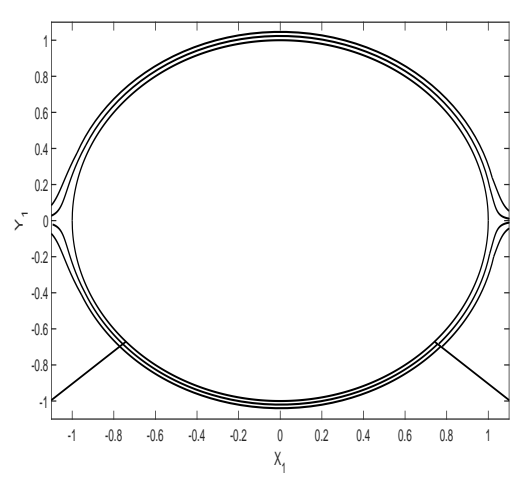

Fig. 4 Simulation of the vector field on the blown-up object in the phase plane $\varepsilon=0$ and $\lambda_{1}=0$. Simulations have been made in each chart and then the trajectories have been mapped onto the blown up object. The full circle is the equator of the hemisphere

Putting all the charts together and mapping the results onto the blown up object allows to understand the dynamics around the hemisphere. This is actually equivalent to the dynamics of the initial system (3) around the origin. The result is illustrated on Figure 4.

We now need to analyze the dynamics for positive and small $\varepsilon$. This is done by using the charts $\left\{\varepsilon_{1}=1\right\}$ with different values of $\lambda_{1}$ around 0 . In chart $\left\{\varepsilon_{1}=1\right\}$, and after division by $r$, the vector 
field around the hemisphere is provided by:

$$
\begin{aligned}
\frac{d X_{1}}{d t} & =\frac{1-b}{2}\left(\lambda_{1} X_{1}-m \frac{1+b}{1-b} Y_{1}-X_{1}^{2}\right)+O(r) \\
\frac{d Y_{1}}{d t} & =\frac{(1+b) b}{2} X_{1}+O(r)
\end{aligned}
$$

(A)

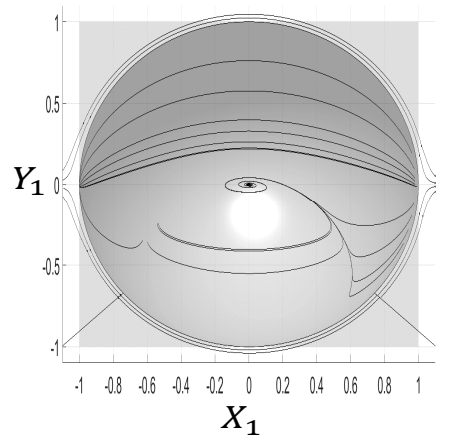

(B)

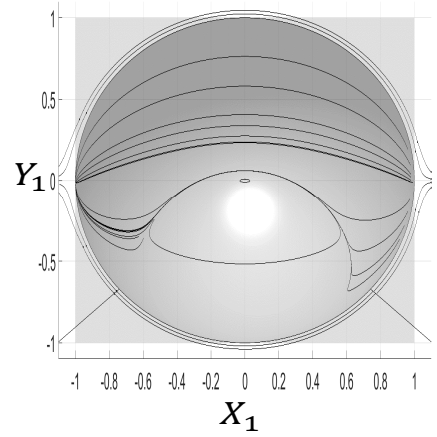

(C)

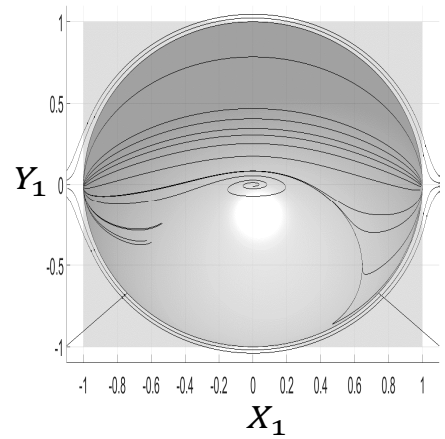

Fig. 5 Illustration of the dynamics induced by the vector field on the blown up object for three values of $\lambda_{1}$ in the chart $\left\{\varepsilon_{1}=1\right\}$. In panel $(\mathrm{A}), \lambda_{1}<0,0$ is a stable equilibrium attracting trajectories initiated under the parabola $\mathcal{P}$, while trajectories initiated above this parabola leave the hemisphere by the West point. In panel $(\mathrm{B}), \lambda_{1}=0$, the origin is a centre. All trajectories initiated below the parabola $\mathcal{P}$ are closed curves surrounding the origin. The trajectories initiated above the parabola leave the hemisphere by the West point. The parabola is invariant under the flow. In panel $(\mathrm{C}), \lambda_{1}>0$ and the origin is an unstable focus. All trajectories leave the hemisphere by the West point. The parameter values used for these simulations are $b=0.1, m=1$ and $\lambda_{1}=-0.1$ in $(\mathrm{A}), \lambda_{1}=0$ in $(\mathrm{B})$ and $\lambda_{1}=0.1$ in $(\mathrm{C})$.

The dynamics on the hemisphere is obtained by setting $r=0$ in the previous system. Let us start with $\lambda_{1}=0$. The origin $\left(X_{1}, Y_{1}\right)=(0,0)$ is the only equilibrium and the vector field is symmetric with respect to the vertical axis of coordinates $X_{1}=0$. The origin is a centre, as we will see by exhibiting a Lyapunov function. Moreover, the parabola $\mathcal{P}$ defined by $Y_{1}=$ $-\frac{1-b}{m(1+b)} X_{1}^{2}+\frac{b(1+b)}{2(1-b)}$ is invariant under the flow. And when $X_{1} \sim \pm \infty$, the parabola equation is equivalent to $Y_{1}=-\frac{1-b}{m(1+b)} X_{1}^{2}$ and indeed it collapses at infinity on the straight lines found in the previous charts associated to this parabola. The dynamics is illustrated on Figure 5.

We define the Hamiltonian function $H$ as follows :

$$
H\left(X_{1}, Y_{1}\right)=\exp \left(\frac{2(1-b)}{b(1+b)} Y_{1}\right)\left(X_{1}^{2}+\frac{m(1+b)}{1-b} Y_{1}-\frac{m b(1+b)^{2}}{2(1-b)^{2}}\right)
$$

This function vanishes on the parabola $\mathcal{P}$ and is positive below the parabola. The level curves of $H$ correspond to trajectories of system (11) when $\lambda_{1}=0$. Let denote by $\gamma$ the trajectory on the hemisphere, it connects the stable branch $\mathcal{M}_{20}^{S}$ to the unstable branch $\mathcal{M}_{20}^{U}$ on the equator of the hemisphere. Along this trajectory, $H$ remains equal to 0 . Let us parameterize $\gamma$ as a function of time. 
Lemma 1 The curve $\gamma$ can be parameterized as follows:

$$
\gamma(t)=\left(\begin{array}{c}
t \\
-\frac{1-b}{m(1+b)} t^{2}+\frac{b(1+b)}{2(1-b)}
\end{array}\right)
$$

The proof of the lemma is straightforward since all $\left(X_{1}(t), Y_{1}(t)\right)$ belonging to $\gamma$ satisfy the equation of $\mathcal{P}$, thus $H(\gamma(t))=0$. The theorem claims that for all small $\varepsilon>0$, there exists a value of $\lambda$ such that the unstable manifold and stable manifold are connected. Actually, the connection will be established from $\gamma$. We first denote by $\overline{\mathcal{M}}_{2 \varepsilon}^{S}\left(\right.$ resp. $\overline{\mathcal{M}}_{2 \varepsilon}^{U}$ ) the stable (resp. unstable) branch of the invariant manifold in the chart $\left\{\varepsilon_{1}=1\right\}$. We will then prove that for all sufficiently small $\varepsilon$, there exists a value of $\lambda$ for which the distance between these manifolds vanishes. We need other notations for expressing this distance, which will follow from the expansion of the differential system (11) in chart $\left\{\varepsilon_{1}=1\right\}$ to order 1 in $r$. One gets:

$$
\begin{aligned}
\frac{d X_{1}}{d t} & =\frac{1-b}{2}\left(-m \frac{1+b}{1-b} Y_{1}-X_{1}^{2}\right)+\frac{1-b}{2} \lambda_{1} X_{1}-r\left(X_{1}^{3}+\frac{m(1+b)}{1-b} X_{1} Y_{1}\right)+O\left(r\left(\lambda_{1}+r\right)\right) \\
\frac{d Y_{1}}{d t} & =\frac{(1+b) b}{2} X_{1}+O\left(r\left(\lambda_{1}+r\right)\right)
\end{aligned}
$$

The right hand-side of this system will be denoted as follows:

$$
F\left(X_{1}, Y_{1}, r, \lambda_{1}\right)=F_{0}\left(X_{1}, Y_{1}\right)+r F_{1, r}\left(X_{1}, Y_{1}\right)+\lambda_{1} F_{1, \lambda_{1}}\left(X_{1}, Y_{1}\right)+O\left(r\left(\lambda_{1}+r\right)\right)
$$

with

$$
\begin{aligned}
F_{0}\left(X_{1}, Y_{1}\right) & =F\left(X_{1}, Y_{1}, 0,0\right) \\
F_{1, r}\left(X_{1}, Y_{1}\right) & =\frac{\partial F}{\partial r}\left(X_{1}, Y_{1}, 0,0\right) \\
F_{1, \lambda_{1}}\left(X_{1}, Y_{1}\right) & =\frac{\partial F}{\partial \lambda_{1}}\left(X_{1}, Y_{1}, 0,0\right)
\end{aligned}
$$

In order to calculate the distance between the stable and unstable branch of the invariant manifold in the chart $\left\{\varepsilon_{1}=1\right\}$, we calculate the deviation of the value taken by $H(t)$ along the whole curve $\gamma$ for every $\left(r, \lambda_{1}\right) \simeq(0,0)$. The distance between $\overline{\mathcal{M}}_{2 \varepsilon}^{S}$ and $\overline{\mathcal{M}}_{2 \varepsilon}^{U}$ is:

$$
\delta\left(r, \lambda_{1}\right)=\int_{H(\gamma(-\infty))}^{H(\gamma(+\infty))} d H(\gamma(t))=\int_{-\infty}^{+\infty} \frac{d H(\gamma(t))}{d t} d t
$$

Now, we can write $\frac{d H}{d t}=\nabla H \cdot F=\nabla H \cdot\left(F_{0}+r F_{1, r}+\lambda_{1} F_{1, \lambda_{1}}+O\left(r\left(\lambda_{1}+r\right)\right)\right.$ and since $H$ is a first integral of the vector field with $r=0$ and $\lambda_{1}=0$, then $\nabla H \cdot F_{0}=0$. It follows:

$$
\delta\left(r, \lambda_{1}\right)=\alpha_{r} r+\alpha_{\lambda_{1}} \lambda_{1}+O\left(r\left(r+\lambda_{1}\right)\right)
$$

where $\alpha_{r}=\int_{-\infty}^{+\infty} \nabla H(\gamma(t)) \cdot F_{1, r}(\gamma(t)) d t$ and $\alpha_{\lambda_{1}}=\int_{-\infty}^{+\infty} \nabla H(\gamma(t)) \cdot F_{1, \lambda_{1}}(\gamma(t)) d t$.

Lemma 2 There exists a function $\lambda_{1 c}$ depending on $r$ in a neighborhood of 0 such that $\delta\left(r, \lambda_{1 c}(r)\right)=$ 0 .

This lemma proves the existence of canard solutions. Its own proof is based on the implicit function theorem. Since $\delta(0,0)=0$, we just need to show that $\frac{d \delta}{d \lambda_{1}}(0,0) \neq 0$, or with the above notations that $\alpha_{\lambda_{1}} \neq 0$.

Using the previous expression for $\alpha_{\lambda_{1}}$ and replacing $\gamma(t)$ by its time parametrization, one gets:

$$
\alpha_{\lambda_{1}}=e(1-b) \int_{-\infty}^{+\infty} \exp \left(-A t^{2}\right) t^{2} d t
$$


where $A=\frac{2(1-b)^{2}}{m b(1+b)^{2}}$ and after integration by parts, we obtain $\alpha_{\lambda_{1}}=\frac{e(1-b)}{2 A} \sqrt{\frac{\pi}{A}} \neq 0$, thus the Implicit Functions theorem applies and lemma (2) is proved.

In order to complete the proof of theorem (1), we use equation (17) where the distance vanishes, thus:

$$
\lambda_{1 c}(r)=-\frac{\alpha_{r}}{\alpha_{\lambda_{1}}} r+O\left(r^{2}\right)
$$

For calculating $\alpha_{r}$, we proceed as for $\alpha_{\lambda_{1}}$ and on gets $\alpha_{r}=-\frac{e}{A^{2}} \sqrt{\frac{\pi}{A}}$ where $A$ is defined as previously, thus:

$$
\lambda_{1 c}(r)=\frac{m b(1+b)^{2}}{(1-b)^{3}} r+O\left(r^{2}\right)
$$
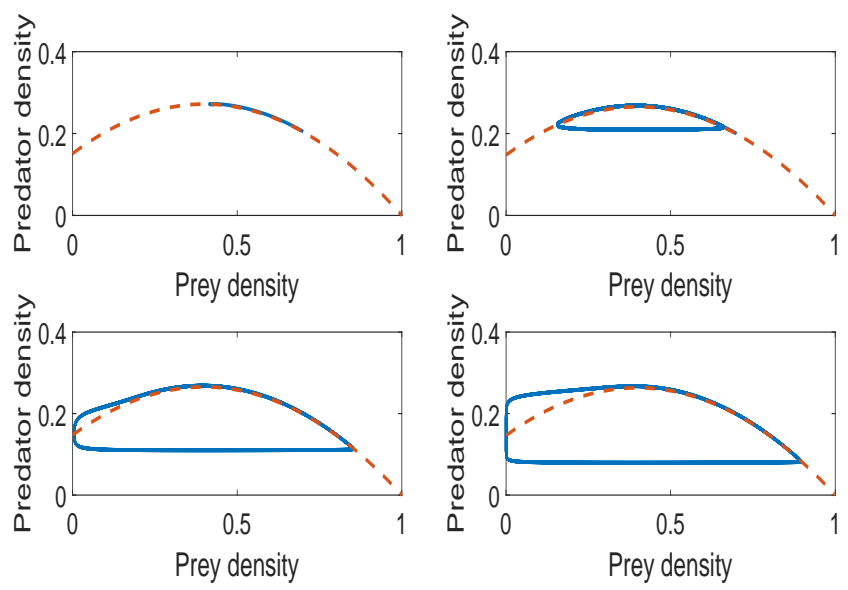

Fig. 6 Several phase portraits showing the birth of the limit cycle in the Hopf bifurcation with a canard phenomenon. On the top left panel, an equilibrium point close to the top of the parabola is globally asymptotically stable. On the top right panel, just after the Hopf bifurcation, a small limit cycle is present. On the bottom left panel, the limit cycle amplitude is drastically enlarged (canard explosion) while the bifurcation parameter is still very close to the value used on the top right panel. Note that the trajectory remains along the unstable branch of the parabola during a finite time. On the bottom right panel, the limit cycle is the largest one. The parameter values used for these simulations are $b=0.2, m=0.9$ and $\lambda=-0.05$ in top-left, $\lambda=0.0112$ in top-right, $\lambda=0.01123$ in bottom-left and $\lambda_{1}=0.03$ in bottom-right. For each panel, $\varepsilon=0.02$.

Finally, since $r=\sqrt{\varepsilon}$ and $\lambda=\sqrt{\varepsilon} \lambda_{1}$, we conclude:

$$
\lambda_{c}(\sqrt{\varepsilon})=\frac{m b(1+b)^{2}}{(1-b)^{3}} \varepsilon+O\left(\varepsilon^{3 / 2}\right)
$$

This proves theorem (1).

\subsection{Numerical simulations}

In Figure 6 the phase portraits are shown for various values of the bifurcation parameter $\lambda$ and a fixed $\varepsilon=0.02$ value. These numerical simulations illustrate the canard explosion phenomenon where for the intermediate parameter values the small limit cycle disappears suddenly (between $\lambda=0.0112$ and $\lambda=0.01123$ ) and is replaced by a big limit cycle. Similar plots with smaller $\varepsilon$-values indicate that the canard explosion occurs for smaller, but positive, $\lambda$ values and furthermore that the transition becomes also sharper. That is, the shape of the limit cycles in the phase-space is closer to that of the relaxation oscillations. These characteristic cycles consist of two fast horizontal episodes and two slow episodes, one downwards along the vertical axis and one upwards along the branch of the parabola. 


\subsection{Comparison of formula (19) with formula (47) in [24]}

We now compare our result in formula (19) with the result we obtained with a different approach in formula (47) in [24]. In that paper, Rosenzweig-MacArthur model with two time scales is also analyzed by using asymptotic expansions of the invariant manifolds. Since the functional response of the predator-prey model is not exactly of the same form, we remind here the algebraic relationship between the parameters of the model in [24], namely $a_{1}, b_{1}, d$ and $\varepsilon$ and the parameters of the present paper, namely $a, b, m$ and $\varepsilon$, the relation is $\left(a_{1}, b_{1}, d, \varepsilon\right)=(a / b, 1 / b, m, \varepsilon)$.

Since we define here $\lambda$ by $\lambda=1-b-2 x_{E}$, by replacing $a$ and $b$ by their expression with respect to $a_{1}$ and $b_{1}$ and considering that in [24] the assumptions $a_{1}=\frac{5}{3} b_{1}$ and $d=1$ have been made, one gets that $b_{1}=\frac{4}{1-\lambda}$ and since $\lambda \simeq 0$ then $b_{1}=4+4 \lambda+O\left(\lambda^{2}\right)$.

If we replace $\lambda$ by $\lambda_{c}$ (formula 19) we obtain $b_{1}=4+4 \frac{\left(1+b_{1}\right)^{2}}{\left(b_{1}-1\right)^{3}} \varepsilon+\ldots$ and taking $b_{1}=4+O(\varepsilon)$ as in [24], this gives:

$$
b_{1}=4+\frac{100}{27} \varepsilon+\ldots
$$

This is exactly the first order term found in formula (47) in [24]. Observe that here the expansion is in $\sqrt{\varepsilon}$ while in [24] it was in $\varepsilon$. Because the $\sqrt{\varepsilon}$-term is missing and the first term is the $(\sqrt{\varepsilon})^{2}=\varepsilon$ term in (19) makes this comparison possible. Note that in [27] it is mentioned that the expansion is a power series in $\varepsilon$ and that is related to a time reversal symmetry property of the blow-up transformation.

\section{Discussion and conclusion}

In this paper, the Rosenzweig-MacArthur model with two time scales has been studied to show that a maximal canard solution exists for small $\varepsilon$. The proof is based on a blow-up method which could allow us to build the canard solution explicitly. A relation between the Hopf bifurcation parameter $\lambda$ and the small parameter $\varepsilon$ has been determined and it permits to get an approximation of $\lambda$ for which the canard solutions arise.

Once our results have been demonstrated, we proposed a comparison with what has been obtained in our previous work [24], showing that both methods provide similar results at the first order in the asymptotic expansion with respect to $\varepsilon$. Since the relation obtained here is a function of $\sqrt{\varepsilon}$ while the relation obtained in our previous work is a function of $\varepsilon$, we can't easily push the comparison further.

Note that the range of values for $\lambda$ in Figure 6 is very small. Moreover, between panel topright $(\lambda=0.0112)$ and panel bottom-left $(\lambda=0.01123)$ the change in $\lambda$ value is very small. This corresponds to a canard explosion, the shape of the canard solution forming the limit cycle in the first case is very different that the shape in the second case, the amplitude of the limit cycle drastically increased between these simulations. This is a very well known phenomenon and this is why finding such canard solution is not obvious if we do not know for which parameter value it occurs. Relations like formula (19) in our paper, or like formula (47) in [24] are therefore useful.

The bifurcation pattern of the Rosenzweig-MacArthur model is simple. For interior steady states either a globally stable equilibrium or limit cycle takes place. For $\varepsilon>0$ the Hopf-theorem applies and shows how the limit cycle emanates at the Hopf bifurcation point where $\lambda=0$ which occurs at the top $S_{T}$ of the prey isocline being a parabola in the phase space. For $\lambda<0$ we have an equilibrium because the Quasi-Steady State Assumption (QSSA) approach is valid where this equilibrium point is closely approached along the stable branch of the parabola.

On the other hand, when $\lambda>0$ the equilibrium is on the unstable branch. Then in the limiting case where $\varepsilon \ll 1$ the shape of the emerging limit cycle is, however, not the well-know cycle but it is distorted by the difference in time scales for the dynamics in the fast prey (horizontal) and slow predator (vertical) direction leading to a relaxation oscillation. Therefore the classical Hopf 
bifurcation analysis has to be replaced by a blow-up analysis as performed in this paper. In this analysis the top $S_{T}$ of the parabola acts as a fold bifurcation. The blow-up technique reveals that the limit cycles follows the initially unstable branch of the parabola which in the blow-up setting is stable in a restricted range for the top $S_{T}$. The shape of the limit cycle looks like a top part of the parabola at the top and a horizontal line at the bottom. For small $\varepsilon$-values these effects occur and remain visible also for larger values of $\varepsilon$ as well as bifurcation increasing $\lambda$-values until it give rise to the canard explosion (see Figure 6) where the small limit cycle disappears abruptly within a small $\lambda$ parameter range.

In [27] also the blow-up technique is used to study the canard phenomenon. Like the approach in this paper they follow the work on blow-up theory in [10] and [11]. Our approach is similar to that used in those references.

Interestingly, the method is general and can be applied to other models. For instance, in [1] the modified Leslie-Gower predator-prey model where the prey reproduces much faster than the predator, is studied. It is stated that for the slow-fast system, the dynamics near the top of the predator parabola isocline needs not to be analyzed with respect to canard explosions. However, numerical simulations (not shown here) show that there is a canard explosion very similar to that observed here for the Rosenzweig-MacArthur predator-prey model shown in Figure 6. Blow-up methods could extend the results provided in [1].

Aknowledgements - The authors aknowledge two anonymous referees and Odo Diekmann for the constructive comments and suggestions which helped us to improve the manuscript.

\section{References}

1. B. Ambrosio, M. A. Aziz-Alaoui, R. Yafia, Canard phenomenon in a slow-fast modified Leslie-Gower model, Math. Biosc. 295 (2018), pp. 48-54

2. P. Auger, R. Bravo de la Parra, J.-C. Poggiale, E. Sanchez, L. Sanz, Aggregation methods in dynamical systems and applications in population and community dynamics, Phys. Life Rev. 5 (2008) pp. 79105

3. P. Auger, B.W.Kooi, R. Bravo de la Parra, J.-C. Poggiale, Bifurcation analysis of a predator-prey model with predators using hawk and dove tactics, J. Theor. Biol., 238, (2006), pp. 597-607

4. E. Benoit, J.L. Callot, F. Diener, M. Diener Chasse au canard Coll. Math. 32, (1981), pp. 37-119

5. H. Boudjellaba, T. SARi Dynamical transcritical bifurcations in a class of predator-prey models, J.Differential Equations 246 (2009), pp. 2205-2225.

6. M. BRøNs, R. KAASEN Canards and mixed-mode oscillations in a forest pest model, Theoretical Population Biology, 77, (2010), pp. 238-242

7. F. Cordoleani, D. Nérini, A. Morozov, M. Gauduchon, J.-C. Poggiale Scaling up the predator functional response in heterogeneous environment: when Holling type III can emerge?, J. Theor. Biol., 336, (2013), pp. 200-208

8. B. DenG, Food chain chaos due to jonction-fold point, Chaos, Vol. 11, (3), (2001), pp.514-525

9. B. Deng, G. Hines, Food chain chaos due to Shilnikov's orbit, Chaos, Vol. 12, (3), (2002), pp.533-538

10. F. Dumortier AND R. Roussarie, Geometric singular perturbation theory beyond normal hyperbolicity, in Multiple Time Scale Dynamical Systems, (2000), Edt. C.K.R.T. Jones and A.I. Khibnik, Springer-Verlag, IMA 122.

11. F. Dumortier And R. Roussarie, Canard cycles and Center Manifolds, Memoirs of the American Mathematical Society, 121, number 577 (1996)

12. N. FEnichel, Persistence ans smoothness of invariant manifolds for flows, Indiana Univ. Math. J., 21 (1971), pp.193-226

13. N., FEnICHeL, Geometric singular perturbation theory for ordinary differential equation, J. Diff. Eq. 31, (1979), pp. 53-98.

14. O. DE FEO, S. RinAldi, Singular homoclinic bifurcations in tritrophic food chains, Math. Biosc., 148, (1998), pp. $7-20$

15. E.H. Flach, S. Schnell, Use and abuse of the Quasi-Steady-State Approximation, IEE Proceedings - Systems Biology, 153, (2006), pp.187-191

16. J.-P. Françoise, C. Piquet, A. Vidal, Enhanced delay bifurcation, Bull. Belg. Math. Soc. Simon Stevin 15 (2008), 825-831.

17. A.HAstings, Timescales and the management of ecological systems, P.N.A.S, 113, (2016), pp. 1456814573

18. M.W. Hirsch, C.C. Pugh, M. Shub, Invariant Manifolds, Lectures Notes in Mathematics, 583, SpringerVerlag, (1977)

19. G. HEK Geometric singular perturbation theory in biological practice, J. Math. Biol. 60 (2010) pp. 347386.

20. F. Hoppensteadt Stability in Systems with Parameter., Trans. Am. Math. Soc. 123, (1966), pp. $521-535$.

21. S.B. Hsu On global stability of a predator - prey system, Math. Biosc., 39, (1978), pp. 1-10 
22. H. Hu, J. Shen, Z. Zhou, Z. Ou Relaxation Oscillations in Singularly Perturbed Generalized Liénard Systems with Non-Generic Turning Points, Mathematical Modelling and Analysis, 22, (2017), pp. 389-407.

23. C.K.R.T. Jones, Geometric singular perturbation theory, in Dynamical systems, Montecatini Terme, Lecture Notes in Mathematics, R. Johnson, ed., Springer-Verlag, 1609 (1994), pp.44-118.

24. B.W. Kooi, J. C. Poggiale Modelling, singular perturbation and bifurcation analyses of bitrophic food chains Math. Biosc. 301, (2018), pp. 93110

25. B.W. Kooi, J.C. Poggiale, P. Auger, S.A.L.M Kooijman Aggregation methods in food chains with nutrient recycling, Ecol. Model., 157, (2002), pp. 6986

26. M. Krupa, P. Szmolyan, Relaxation oscillation and canard explosion, J. Differ. Equ. 174, (2001), pp. 312368.

27. M. Krupa, P. Szmolyan, Extending geometric singular perturbation theory to nonhyperbolic points-fold and canard points in two dimensions, SIAM J. Math. Anal., 33 (2001), pp.286-314.

28. M. KRupa, P. Szmolyan, Extending slow manifolds near transcritical and pitchfork singularities, Nonlinearity 4 (2001), pp. 1473-1491.

29. C. KueHn, Multiple time scale dynamics, Applied Mathematical Sciences, 191, (2015), Springer

30. S. LEVIN Scale and Predictability in Ecological Modeling in Modeling and Management of Resources under Uncertainty, (1985), Edt. T.L. Vincent, Y. Cohen, W.J. Grantham, G.P. Kirkwood, J.M. Skowronski, Lect. Notes Biomath., 72, pp. 2-10.

31. C. Li, H. ZHu, Canard cycles for predator - prey systems with Holling types of functional response, J.Diff. Eq. 254, (2013), pp. 879-910

32. N. Mehidi, T. SARI, Limit cycles of a food chain system, Proceedings of Pau Congress, (1992)

33. N. Menidi, A prey - predator - superpredator system, Journal of Biological Systems, Vol. 9, (3), (2001), pp. $187-199$

34. S. Muratori, S. Rinaldi, Low- and high-frequency oscillations in three-dimensional food chain systems, SIAM J. Appl. Math., Vol. 52, (6), (1992), pp. 1688-1706

35. J.-C. Poggiale, P. Auger, Impact of spatial heterogeneity on a predatorprey system dynamics, C. R. Biologies, 327, (2004), pp. 10581063.

36. J.-C. Poggiale, P. Auger, Fast oscillating migrations in a predator - prey model, Math. Mod. and Meth. in App. Sc., 6, (1996), pp. 217-226.

37. J.C. Poggiale, Predator-Prey Models in Heterogeneous Environment : Emergence of Functional Response, Math. Comp. Model., 27, (1998), pp. 63-71

38. J.-C. Poggiale, M. Gauduchon and P. Auger Enrichment Paradox Induced by Spatial Heterogeneity in a Phhytoplankton - Zooplankton System, Math. Mod. Natur. Phen., 3, (2008), pp. 87-102

39. J.-C. Poggiale, P. Auger, F. Cordolani, T. Nguyen-Hud, Study of a virus-bacteria interaction model in a chemostat: application of geometrical singular perturbation theory, Phil. Trans. R. Soc. A, 367, (2009), pp. 4685-4697

40. A. Pokrovskit, E. Shchepakina, V. Sobolev Canard Doublet in a Lotka-Volterra type model, Journal of Physics: Conference Series 138 (2008), doi:10.1088/1742-6596/138/1/012019

41. S. Rinaldi, S. Muratori, Slow-fast limit cycles in predator-prey models, Ecological Modelling, 61, (1992), pp. $287-308$

42. M.L. Rosenzweig, Paradox of enrichment: destabilization of exploitation ecosystems in ecological time, Science 171 (1971) pp. 385387.

43. S. SAdhu, Canards and Mixed-Mode Oscillations in a Singularly Perturbed Two Predators-One Prey Model, Proceedings of Dynamic Systems and Applications, 7, (2016), pp. 211219

44. K. Sakamoto, Invariant manifolds in singular perturbation problems for ordinary differential equations, Proc. Royal Soc. Edinburgh, Vol. 116A, (1992), pp. 45-78

45. M. Schauer, R. Heinrich Quasi-Steady-State Approximation in the Mathematical Modeling of Biochemical Reaction Networks Math. Biosc. 65, (1983), pp. 155-170

46. S.K. Shoffner, S. SChNell Approaches for the estimation of timescales in nonlinear dynamical systems: Timescale separation in enzyme kinetics as a case study Math. Biosc. 287 (2017), pp. 122129

47. A.N. TIKHONOv Systems of differential equations containing small parameters in the derivatives., Mat. Sb. 31 (1952), pp. 575-586.

48. M. VIDYASAGAR Decomposition techniques for large-scale systems with nonadditive interactions: stability and stabilizability, IEEE Trans. Autom. Cont. 25, (1980), pp. 773-779.

49. A. Vidal, J.-P. Francoise Canard cycles in global dynamics, Int. J. Bif. Chaos, 22, (2012)

50. S. Wiggins, Normally hyperbolic invaraint manifolds in dynamical systems, App. Math. Sc. Series,SpringerVerlag, 105 (1994)

\section{Appendix}

In this appendix, we prove that a canard solution exists in the vicinity of the point $S_{C}$ located at the intersection of the parabola and the vertical axis in the Rosenzweig-MacArthur model. Let us start with system (1):

$$
\begin{aligned}
& \frac{d x}{d t}=x\left(b+(1-b) x-a y-x^{2}\right) \\
& \frac{d y}{d t}=\varepsilon((a-m) x-m b) y
\end{aligned}
$$


The point $S_{C}$ corresponds to a point where the fast dynamics exhibits a transcritical bifurcation when the slow variable $y$ decreases: for $y>\frac{b}{a}, x=0$ is stable for the fast equation while for $y>\frac{b}{a}, x=0$ is unstable for the fast equation. In other words, $S_{C}$ is at the intersection of the invariant manifolds $\mathcal{M}_{10}$ (the $y$-axis) and $\mathcal{M}_{20}$ (the parabola). As in the article, by adding $\frac{d \varepsilon}{d t}=0$ to system (20), we get a 3 -dimensional system. By Fenichel's theorem, $\mathcal{M}_{10}$ and $\mathcal{M}_{20}$ persist for small positive $\varepsilon$ at each point where they are normally hyperbolic. It is not the case in $S_{C}$. In [5], [16], [28] and [49] for instance, the authors address the problem of this type of singularity. Our case is not considered in [28].

Again, the blow-up replaces the point $S_{C}$ by a geometrical object (a hemisphere here) on which all points are non singular (the normal hyperbolicity is reached). Even if it is quite obvious in this example, one will see that the stable branch of $\mathcal{M}_{10}$ is connected to the unstable branch of $\mathcal{M}_{10}$ by a trajectory of the blown-up vector field, this is actually due to the fact that the vertical axis is invariant for the complete system (20). This connection leads to a canard solution. In this case, the canard solution gives birth to a so-called delayed bifurcation (see [16], [5] and [49]), those works do not use blow-up method for their analysis.

We translate the point $S_{C}$ at the origin with the following change of coordinates $(X, Y)=$ $(x, y-b / a)$, the new system reads:

$$
\begin{aligned}
\frac{d X}{d t} & =X\left((1-b) X-a Y-X^{2}\right) \\
\frac{d y}{d t} & =\varepsilon\left(Y+\frac{b}{a}\right)((a-m) X-m b)
\end{aligned}
$$

In order to study the singularity, where the normal hyperbolicity is lost, we use the following blow-up:

$$
(X, Y, \varepsilon)=\left(r X_{1}, r Y_{1}, r^{2} \varepsilon_{1}\right)
$$

where $X_{1}^{2}+Y_{1}^{2}+\varepsilon_{1}^{2}=1$ and $r \in\left[0, r_{0}\right)$ with $r_{0}>0$. As explained in the article, we study the dynamics by using charts.

In chart $\left\{X_{1}=1\right\}$, we have : $X=r, Y=r Y_{1}$ and $\varepsilon=r^{2} \varepsilon_{1}$, and after division by $r$ for desingularization, the system reads:

$$
\begin{aligned}
\frac{d r}{d t} & =r\left(1-b-a Y_{1}\right)+O\left(r^{2}\right) \\
\frac{d Y_{1}}{d t} & =\varepsilon_{1} \frac{b^{2} m}{a}-Y_{1}\left(1-b-a Y_{1}\right)+O(r) \\
\frac{d \varepsilon_{1}}{d t} & =-2 \varepsilon_{1}\left(1-b-a Y_{1}\right)+O(r)
\end{aligned}
$$

In chart $\left\{Y_{1}=1\right\}$, we have : $X=r X_{1}, Y=r$ and $\varepsilon=r^{2} \varepsilon_{1}$, and after division by $r$ for desingularization, the system reads:

$$
\begin{aligned}
\frac{d r}{d t} & =-r \varepsilon_{1} \frac{m b^{2}}{a}+O\left(r^{2}\right) \\
\frac{d X_{1}}{d t} & =X_{1}\left((1-b) X_{1}-a+\varepsilon_{1} \frac{m b^{2}}{a}\right)+O(r) \\
\frac{d \varepsilon_{1}}{d t} & =2 \varepsilon_{1}^{2} \frac{m b^{2}}{a}+O(r)
\end{aligned}
$$


In chart $\left\{Y_{1}=-1\right\}$, we have : $X=r X_{1}, Y=-r$ and $\varepsilon=r^{2} \varepsilon_{1}$, and after division by $r$ for desingularization, the system reads:

$$
\begin{aligned}
\frac{d r}{d t} & =r \varepsilon_{1} \frac{m b^{2}}{a}+O\left(r^{2}\right) \\
\frac{d X_{1}}{d t} & =X_{1}\left((1-b) X_{1}+a-\varepsilon_{1} \frac{m b^{2}}{a}\right)+O(r) \\
\frac{d \varepsilon_{1}}{d t} & =-2 \varepsilon_{1}^{2} \frac{m b^{2}}{a}+O(r)
\end{aligned}
$$

In chart $\left\{\varepsilon_{1}=1\right\}$, we have : $X=r X_{1}, Y=r Y_{1}$ and $\varepsilon=r^{2}$, and after division by $r$ for desingularization, the system reads:

$$
\begin{aligned}
\frac{d X_{1}}{d t} & =X_{1}\left((1-b) X_{1}-a Y_{1}\right)+O(r) \\
\frac{d Y_{1}}{d t} & =-\frac{m b^{2}}{a}+O(r)
\end{aligned}
$$

Note that since we are not interested in the dynamics of the system for negative values of $X$, we don't use the chart $\left\{X_{1}=-1\right\}$ and focus on the hemishpere contained in $X_{1}>0$.

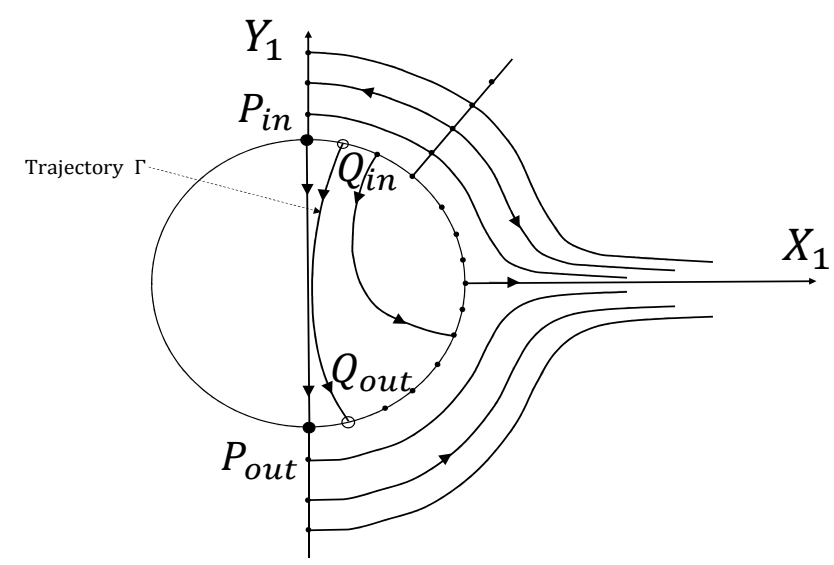

Fig. 7 Blow-up at the point $S_{C}$ for system (1). Note that there is a trjactory connecting $P_{\text {in }}$ to $P_{o u t}$ on the hemisphere $\varepsilon_{1}>0$. This trajectory is in the plane $\left\{\left(X_{1}, Y_{1}, \varepsilon_{1}\right) \mid X_{1}=0, Y_{1}=0\right\}$ and corresponds to the vertical axis of the initial model (1) for $\varepsilon_{1}>0$. Moreover, we represent on this graph a trajectory $\Gamma$ connecting $Q_{i n}$ to $Q_{\text {out }}$ (open circles), which corresponds to a solution which may represent the trace of the limit cycle of system (1) on the hemisphere.

Figure 7 illustrates the result of the blow-up. The disk in the center represents the hemisphere $\left\{\left(X_{1}, Y_{1}, \varepsilon_{1}\right), X_{1}^{2}+Y_{1}^{2}+\varepsilon_{1}^{2}=1, \varepsilon_{1} \geq 0\right\}$. The circle of radius one is the equator of this hemisphere. The remaining part of the diagram corresponds to the plane $\{\varepsilon=0\}$ minus the origin (the point $\left.S_{C}\right)$. In this plane, the dynamics is equivalent to the dynamics of system (21), we focus here to the locus $\left\{X_{1} \geq 0\right\}$. The vertical half straight line above the disk is the stable branch of the invariant manifold $\mathbb{1}_{10}$, the vertical half straight line below the disk is the unstable branch of this invariant manifold. The trajectory between the point $P_{\text {in }}$ and the point $P_{\text {out }}$ connects these branches on the hemisphere, explaining the occurrence of the canard solution. This solution is related to a delayed bifurcation ( [49] or [41] for instance). 